\title{
Metallic-thin-film instability with spatially correlated thermal noise
}

\author{
Javier A. Diez and Alejandro G. González \\ Instituto de Física Arroyo Seco (CIFICEN-CONICET), \\ Universidad Nacional del Centro de la Provicia de Buenos Aires, Pinto 399, 7000, Tandil, Argentina \\ Roberto Fernández \\ Department of Mathematics, Utrecht University, P. O. Box 800103508 TA Utrecht \\ (Received 24 June 2015; revised manuscript received 22 October 2015; published 15 January 2016)
}

\begin{abstract}
We study the effects of stochastic thermal fluctuations on the instability of the free surface of a flat liquid metallic film on a solid substrate. These fluctuations are represented by a stochastic noise term added to the deterministic equation for the film thickness within the long-wave approximation. Unlike the case of polymeric films, we find that this noise, while remaining white in time, must be colored in space, at least in some regimes. The corresponding noise term is characterized by a nonzero correlation length, $\ell_{c}$, which, combined with the size of the system, leads to a dimensionless parameter $\beta$ that accounts for the relative importance of the spatial correlation $\left(\beta \sim \ell_{c}^{-1}\right)$. We perform the linear stability analysis (LSA) of the film both with and without the noise term and find that for $\ell_{c}$ larger than some critical value (depending on the system size), the wavelength of the peak of the spectrum is larger than that corresponding to the deterministic case, while for smaller $\ell_{c}$ this peak corresponds to smaller wavelength than the latter. Interestingly, whatever the value of $\ell_{c}$, the peak always approaches the deterministic one for larger times. We compare LSA results with the numerical simulations of the complete nonlinear problem and find a good agreement in the power spectra for early times at different values of $\beta$. For late times, we find that the stochastic LSA predicts well the position of the dominant wavelength, showing that nonlinear interactions do not modify the trends of the early linear stages. Finally, we fit the theoretical spectra to experimental data from a nanometric laser-melted copper film and find that at later times, the adjustment requires smaller values of $\beta$ (larger space correlations).
\end{abstract}

DOI: 10.1103/PhysRevE.93.013120

\section{INTRODUCTION}

The breakup of a flat thin liquid film on a solid substrate is a fundamental issue in the study of free surface instabilities. The phenomenon is determined by partially understood effects acting at the nanometric scale. These effects can be studied, in some detail, through simulations of molecular dynamics but at the cost of heavy computational resources and severe limitations on the thickness of the films. An alternative approach resorts to stochastic descriptions of relevant intermolecular interactions through appropriate "noisy" hydrodynamical equations. This type of description was pioneered by Landau [1], who proposed additional phenomenological fluctuation terms that were exploited, for instance, by Uhlenbeck and Fox [2] for Brownian particles. The terms were later justified, from the microscopical point of view, as corresponding to a longwave approximation applied to the deterministic Boltzmann equation [3]. The resulting equations have been used in the study of bulk instability phenomena, such as turbulence in randomly stirred fluids [4], Rayleigh-Benard convection [5], and Taylor-Couette flow [6].

In general, approaches based on hydrodynamic NavierStokes equations supplemented by stochastic fluctuation terms have been found to be valid to describe the instability of bulk matter [7] but to fail for thin-film phenomena. This is because the framework does not properly account for the thermal agitation of molecules, known to be relevant for the behavior of open surfaces at small scales [8-10]. The failure is particularly evident in thermally triggered phenomena, such as the breakup of nanojets $[9,11]$ or the glass transition of polymer films [12]. Nevertheless, the continuum hydrodynamic approach can be extended to phenomena driven by thermal agitation by using stochastic differential equations [9]. These equations are obtained by adding a contribution involving a stochastic process or field describing the noise, usually assumed to be uncorrelated (white) noise both in space and time. The lack of correlations in time is associated with the absence of memory effects due to thermal fluctuations. The validity of the hypothesis of no spatial correlation of thermal noise is, in our opinion, less clear.

In this paper, we apply the noisy hydrodynamic approach to study the effect of thermal noise on metallic films laterally much larger (up to microns) than their thicknesses and show that, at least in some regimes, the noise must be considered spatially correlated. Our paper has a double objective: On the one hand, we contribute to the understanding of breakup instabilities in films used in the design of microfluidic devices. On the other hand, we present a case study that shows the limitations of the spatial white noise assumption, together with a slightly generalized mathematical formalism that can be of use in other systems with spatially correlated noise.

Thin-film instabilities have been studied mostly for polymeric films [13-15]. In particular, pattern analysis procedures have been proposed-based in Minkowsky invariants-to compare experiments with theoretical and simulation results for these films [16] and to test whether patterns correspond to a Gaussian field [17]. These procedures show satisfactory agreement between observations and theoretical studies assuming space-time white thermal noise. In contrast, unstable liquid metal films have not been the object of comparably thorough studies. In these films, the solid coating is melted by laser and, since the deposition of energy is not strictly uniform 
throughout the illuminated spot, the thermal fluctuations-and thus the liquid lifetime-may not be the same for all regions. In such a context, thermal correlations can be expected to become spatially extended.

Our paper is a contribution towards filling the gap in the understanding of metallic thin-film breakup. We address the issue at three different levels. At the theoretical level, we propose a stochastic version of the thin-film equationbased on the lubrication approximation for incompressible hydrodynamic equations [8]-with spatially extended noise (see Sec. II). In Sec. III, we perform a linear stability analysis of the film under perturbations with normal modes. This analysis allows us to compare the influence of the correlation length of spatial fluctuations on the spectra of unstable modes. In particular, the amplitudes of these modes are seen to increase with decreasing correlation length, while the wave number of the mode with maximum amplitude can be lower or larger than the deterministic one depending on this length.

In Sec. IV we solve numerically the stochastic thin-film equation and compare the results with the linear solution obtained previously. As expected, fluctuations accelerate breakups and rupture times decrease with the correlation length of the fluctuations. Fourier spectra of profile thickness are reasonably well described by the linear stability predictions both at early and late times. And, finally, in Sec. V we compare the predictions of our stochastic differential equations with experimental Fourier spectra previously obtained [18] from scanning electron microscope (SEM) images of the instability of a melted copper film. We find that optimal fitting is not achieved through white spatial noise; rather, it requires fluctuations of increasing correlation length as the center of the spot is approached (that is, as the liquid lifetime increases).

\section{THIN-FILM EQUATIONS WITH STOCHASTIC NOISE}

In order to somehow include the thermal agitation in the framework of the continuous mechanics, it is considered that the film molecules modify the surface forces that describe the interaction between the fluid inside a volume element and its surroundings. We adopt the lubrication approximation of the stochastic Navier-Stokes equation $[8,19]$ and introduce an additional random symmetric term, $\mathcal{S}$, in the expression of the Newtonian stress tensor. The most relevant component of $\mathcal{S}$ is $\mathcal{S}_{i z}$, where $i$ can be either $x$ or $y$ and indicates a direction parallel to the substrate while $z$ stands for the normal one. These components have zero mean,

$$
\left\langle\mathcal{S}_{i z}(\vec{x}, t)\right\rangle=0,
$$

and correlations

$$
\left\langle\mathcal{S}_{i z}(\vec{x}, t) \mathcal{S}_{j z}\left(\vec{x}^{\prime}, t^{\prime}\right)\right\rangle=2 \mu k_{B} T F\left(\vec{x}-\vec{x}^{\prime}\right) \delta\left(t-t^{\prime}\right) \delta_{i, j},
$$

where $i, j=x, y, \mu$ is the fluid viscosity, $\delta$ is the Dirac delta function, and $\vec{x}=(x, y)$. Here $k_{B}$ and $T$ are the Boltzmann constant and fluid temperature, respectively. $F$ stands for a translation-invariant (generalized) function; the standard choice of spatial white noise corresponds to $F\left(\vec{x}-\vec{x}^{\prime}\right)=$ $\delta\left(\vec{x}-\vec{x}^{\prime}\right)$. The form (2) is consistent with the fluctuationdissipation theorem which relates the fluctuations of physical quantifies to the dissipative properties of the system. The theorem assumes the existence of some form of local equilibrium, hence the resulting hydrodynamical equations are only valid at scales much larger than the molecular scale. This is a further argument in favor of considering functions $F$ with extended support ("colored" space noise). In the same approximation, the pressure terms in the isotropic part of the stress for a film of local thickness $h(\vec{x}, t)$ are given, as usual, by the capillary pressure, $-\gamma \nabla^{2} h$ (where $\gamma$ is the surface tension), and the disjoining-conjoining pressure (van der Waals force), $(h)$. Thus, the reduction of the Navier-Stokes equations under the lubrication approximation leads to [19]:

$$
\begin{aligned}
& 3 \mu \frac{\partial h}{\partial t}+\vec{\nabla} \cdot\left[h^{3} \vec{\nabla}\left(\gamma \nabla^{2} h+\Pi(h)\right)\right] \\
&-\vec{\nabla} \cdot\left[\int_{0}^{h}(h-z) \mathcal{S}_{\| z}(z) d z\right]=0,
\end{aligned}
$$

where $\mathcal{S}_{\| z}=\left(\mathcal{S}_{x z}, \mathcal{S}_{y z}\right)$. Note that the new noise term in Eq. (3), while complicated, has the advantage that it maintains the conservative form of the equation, incorporating a random current which acts as another driving force.

Since we can assume that the process is Markovian, the usual procedure of making a Krammers-Moyal expansion of the master equation and retaining the first significant terms leads to a Fokker-Planck equation that is easier to solve but retains all the meaningful features of the problem [20,21]. The function $h$ is, in fact, a stochastic process whose distribution evolution follows the appropriate Fokker-Planck equation [19], corresponding to the Langevin equation,

$$
3 \mu \frac{\partial h}{\partial t}+\vec{\nabla} \cdot\left[h^{3} \vec{\nabla}\left(\gamma \nabla^{2} h+\Pi(h)\right)\right]-\vec{\nabla} \cdot\left[\sqrt{3 h^{3}} \vec{\xi}(\vec{x}, t)\right]=0,
$$

with a single multiplicative conserved noise vector $\vec{\xi}(\vec{x}, t)$ satisfying [2,19]

$$
\begin{aligned}
\langle\vec{\xi}(\vec{x}, t)\rangle & =0, \\
\left\langle\xi_{i}(\vec{x}, t) \xi_{j}\left(\vec{x}^{\prime}, t^{\prime}\right)\right\rangle & =2 \mu k_{B} T F\left(\vec{x}-\vec{x}^{\prime}\right) \delta\left(t-t^{\prime}\right) \delta_{i, j} .
\end{aligned}
$$

The $\delta$-correlated noise in time ensures that the results of studying of the Fokker-Planck equation are equivalent to those of the Langevin equation [21]. Assuming symmetry along $y$ axis, the one-dimensional version of Eq. (4) for $h(x, t)$ is

$$
3 \mu \frac{\partial h}{\partial t}+\frac{\partial}{\partial x}\left[h^{3}\left(\gamma \frac{\partial^{3} h}{\partial x^{3}}+\frac{\partial \Pi}{\partial x}\right)\right]-\frac{\partial}{\partial x}\left[\sqrt{3 h^{3}} \xi(x, t)\right]=0,
$$

where, for brevity, $\xi(x, t)$ stands for $\xi_{x}(x, t)$.

Since the only characteristic length scale of an infinite film is its thickness, $h_{0}$, we define the following dimensionless variables:

$$
\tilde{x}=\frac{x}{h_{0}}, \quad \tilde{y}=\frac{h}{h_{0}}, \quad \tilde{t}=\frac{t}{t_{0}}, \quad \tilde{\Pi}=\frac{h_{0}}{\gamma} \Pi, \quad \Theta=\frac{\xi}{\sqrt{T} \Theta_{0}},
$$

where the scales of time, $t_{0}$, and noise, $\Theta_{0}$, are to be determined in terms of the characteristic parameters of the problem. Here we take the capillary pressure, $\gamma / h_{0}$, as the scale for the disjoining pressure, and we have considered the temperature dependence of the noise amplitude as given by Eq. (5). Thus, 
the dimensionless version of Eq. (6) is as follows:

$$
\frac{\partial \tilde{h}}{\partial \tilde{t}}+\frac{\partial}{\partial \tilde{x}}\left[\tilde{h}^{3}\left(\frac{\partial^{3} \tilde{h}}{\partial \tilde{x}^{3}}+\frac{\partial \tilde{\Pi}}{\partial \tilde{x}}\right)\right]-\sqrt{2 \sigma} \frac{\partial}{\partial \tilde{x}}\left[\tilde{h}^{3 / 2} \Theta(\tilde{x}, \tilde{t})\right]=0,
$$

where

$$
t_{0}=\frac{3 \mu h_{0}}{\gamma}, \quad \sigma=\frac{k_{B} T}{\gamma h_{0}^{2}}, \quad \Theta_{0}=\gamma \sqrt{\frac{2 \sigma}{3 h_{0}}},
$$

and

$$
\left\langle\Theta(\tilde{x}, \tilde{t}) \Theta\left(\tilde{x}^{\prime}, \tilde{t}^{\prime}\right)\right\rangle=\tilde{F}\left(\tilde{x}-\tilde{x}^{\prime}\right) \delta\left(\tilde{t}-\tilde{t}^{\prime}\right),
$$

with $\tilde{F}=F / h_{0}^{2}$ a dimensionless correlation [for white noise $\tilde{F}(\widetilde{\vec{x}})=\delta(\widetilde{\vec{x}})]$. Note that $\sigma$ measures the relative importance of the stochastic term (thermal noise) with respect to the deterministic part, and it is given by the ratio between the thermal and surface energies of the system. Since typical experimental data yield $\sigma$ of the order of $10^{-4}$ (or even less) we will consider here this parameter within this range of values in order to look for effects on the film instability.

As regards to the form of $\Pi$, we take into account both the attractive and repulsive intermolecular liquid-solid forces, so it includes both the disjoining and conjoining pressure terms in the form [22]

$$
\Pi(h)=\kappa f(h)=\kappa\left[\left(\frac{h_{*}}{h}\right)^{3}-\left(\frac{h_{*}}{h}\right)^{2}\right],
$$

where $h_{*}$ is the dimensional equilibrium thickness and $\kappa$ (with units of pressure) is given by

$$
\kappa=\frac{\mathcal{A}}{6 \pi h_{*}^{3}}
$$

with $\mathcal{A}$ being the Hamaker constant. Alternatively, it is also useful to define $\kappa$ in terms of the contact angle, $\theta$, as [22]

$$
\kappa=\frac{2 \gamma(1-\cos \theta)}{h_{*}} .
$$

In dimensionless variables, $\kappa$ becomes $K=\kappa h_{0} / \gamma$, and then the final version of Eq. (8) is

$$
\begin{aligned}
\frac{\partial h}{\partial t} & +\frac{\partial}{\partial x}\left[h^{3}\left(\frac{\partial^{3} h}{\partial x^{3}}+K f^{\prime}(h) \frac{\partial h}{\partial x}\right)\right]-\sqrt{2 \sigma} \frac{\partial}{\partial x}\left[h^{3 / 2} \Theta(x, t)\right] \\
& =0
\end{aligned}
$$

where we omit the tilde ( $)$ for brevity here and from now on.

As said before, the stochastic term $\Theta(x, t)$ is considered to be white noise with respect to time. Formally, this means that it is of the form

$$
\Theta(x, t)=\frac{\partial W(x, t)}{\partial t},
$$

where, for each $x$, the process $W(x, \cdot)$ is a standard Brownian motion, namely the translation-invariant continuous process with independent increments, each of which is normally distributed:

$$
W(x, t+\Delta)-W(x, t) \sim \mathcal{N}(0, \Delta) .
$$

Here $\mathcal{N}(0, \Delta)$ is a normal distribution with zero mean and variance $\Delta$, and “ " stands for equality of distributions.

\section{LINEAR STABILITY ANALYSIS (LSA) OF THE STOCHASTIC THIN-FILM EQUATION}

\section{A. Linearized equation in Fourier space}

The linearized equation is expected to hold at the beginning of the instability process, when the deviations, $\delta h(x, t)=$ $h(x, t)-\tilde{h}_{0}$, from the initial average film height are small (even if $\tilde{h}_{0}=1$, we keep this notation for clarity). By expanding Eq. (14) up to first order in $\delta h$ and $\Theta$ (assuming that the noise amplitude is small as well) we obtain the linear stochastic equation,

$$
\frac{\partial \delta h}{\partial t}+\tilde{h}_{0}^{3}\left[\frac{\partial^{4} \delta h}{\partial x^{4}}+K f^{\prime}\left(\tilde{h}_{0}\right) \frac{\partial^{2} \delta h}{\partial x^{2}}\right]-\sqrt{2 \sigma \tilde{h}_{0}^{3}} \frac{\partial \Theta}{\partial x}=0 .
$$

It is convenient to look for its solution in the Fourier space, so we use the spatial transform by

$$
\widehat{\delta h}(q, t)=\int_{-\infty}^{\infty} \delta h(x, t) e^{-i q x} d x .
$$

Therefore, Eq. (17) becomes

$$
\frac{\partial \widehat{\delta h}(q, t)}{\partial t}=\omega(q) \widehat{\delta h}(q, t)+i \sqrt{2 \sigma \tilde{h}_{0}^{3}} q \widehat{\Theta},
$$

where we define

$$
\omega(q)=4 \omega_{m}\left[\left(\frac{q}{q_{c}}\right)^{2}-\left(\frac{q}{q_{c}}\right)^{4}\right]
$$

that corresponds to the dispersion relation of the deterministic case [22]. Here

$$
q_{c}=\sqrt{K f^{\prime}\left(\tilde{h}_{0}\right)}, \quad \omega_{m}=\frac{\tilde{h}_{0}^{3} q_{c}^{4}}{4}
$$

are the critical (marginal) wave number and the maximum growth rate, respectively. The wave number of maximum growth rate is

$$
q_{m}=q_{c} / \sqrt{2} .
$$

Since Eq. (19) is an equation of the Langevin type, its solution is given by [23,24],

$$
\widehat{\delta h}(q, t)=e^{\omega(q) t} \widehat{\delta h}(q, 0)+i \sqrt{2 \sigma \tilde{h}_{0}^{3}} q \int_{0}^{t} e^{\omega(q)(t-s)} d \widehat{W}(q, s) .
$$

The process $\widehat{W}(q, \cdot)$ is the primitive Brownian process of the time white noise $\widehat{\Theta}(q, \cdot)$ [see (16)]:

$$
\widehat{\Theta}(q, t)=\frac{\partial \widehat{W}(q, t)}{\partial t} .
$$

By (10), the autocorrelation of the Fourier transformed noise is

$$
\begin{aligned}
& \left\langle\widehat{\Theta}(q, t) \widehat{\Theta}\left(q^{\prime}, t^{\prime}\right)\right\rangle \\
& \quad=\int_{-\infty}^{\infty} \int_{-\infty}^{\infty}\left\langle\Theta(x, t) \Theta\left(x^{\prime}, t^{\prime}\right)\right\rangle e^{-i q x} e^{-i q^{\prime} x^{\prime}} d x d x^{\prime} \\
& \quad=\int_{-\infty}^{\infty} \int_{-\infty}^{\infty} \delta\left(t-t^{\prime}\right) F\left(x-x^{\prime}\right) e^{-i\left(q x+q^{\prime} x^{\prime}\right)} d x d x^{\prime} \\
& =2 \pi \delta\left(q+q^{\prime}\right) \delta\left(t-t^{\prime}\right) \widehat{F}(q),
\end{aligned}
$$


where

$$
\widehat{F}(q)=\int_{-\infty}^{\infty} F(u) e^{-i q u} d u
$$

is the Fourier transform of the correlation function $F$ and we have applied the identity $\int_{-\infty}^{\infty} e^{-i q x} d x=2 \pi \delta(q)$. From (24) and (26) we obtain that $\widehat{W}$ has autocorrelations

$$
\left\langle\widehat{W}(q, t) \widehat{W}\left(q^{\prime}, t^{\prime}\right)\right\rangle=2 \pi \delta\left(q+q^{\prime}\right) \widehat{F}(q)\left(t \wedge t^{\prime}\right),
$$

where $t \wedge t^{\prime}$ stands for the minimum of $t$ and $t^{\prime}$. Here the symbol $\wedge$ is employed as it is usual in mathematics since it is consistent with the set theoretical symbol for intersections (see, e.g., Ref. [25]).

To study the instability evolution in the spectral space we calculate the autocorrelation

$$
\left\langle\widehat{\delta h}(q, t) \widehat{\delta h}\left(q^{\prime}, t^{\prime}\right)\right\rangle=C_{1}+C_{2}+C_{3}+C_{4},
$$

where the terms on the right-hand side are defined as follows:

$$
\begin{gathered}
C_{1}=\left\langle\widehat{\delta h}(q, 0) \widehat{\delta h}\left(q^{\prime}, 0\right)\right\rangle e^{\omega(q) t} e^{\omega\left(q^{\prime}\right) t^{\prime},} \\
C_{2} \propto\left\langle\widehat{\delta h}(q, 0) d \widehat{W}\left(q^{\prime}, t^{\prime}\right)\right\rangle, \\
C_{3} \propto\left\langle\widehat{\delta h}\left(q^{\prime}, 0\right) d \widehat{W}(q, t)\right\rangle, \\
C_{4}=-2 \sigma \tilde{h}_{0}^{3} q^{2}\left\langle\int_{0}^{t} e^{\omega(q)(t-s)} d \widehat{W}(q, s)\right. \\
\left.\times \int_{0}^{t^{\prime}} e^{\omega\left(q^{\prime}\right)\left(t^{\prime}-s^{\prime}\right)} d \widehat{W}\left(q^{\prime}, s^{\prime}\right)\right\rangle .
\end{gathered}
$$

To calculate $C_{1}$ we determine the initial height-height correlation:

$$
\begin{aligned}
\left\langle\delta \widehat{h}(q, 0) \delta \widehat{h}\left(q^{\prime}, 0\right)\right\rangle= & \int_{-\infty}^{\infty} \int_{-\infty}^{\infty}\left\langle\delta h(x, 0) \delta h\left(x^{\prime}, 0\right)\right\rangle \\
& \times e^{-i q x} e^{-i q^{\prime} x^{\prime}} d x d x^{\prime} \\
= & \int_{-\infty}^{\infty} \int_{-\infty}^{\infty} F_{0}(u) e^{-i q u} e^{-i\left(q+q^{\prime}\right) x^{\prime}} d x^{\prime} d u \\
= & 2 \pi \widehat{F}_{0}(q) \delta\left(q+q^{\prime}\right) .
\end{aligned}
$$

Hence,

$$
C_{1}=2 \pi \widehat{F}_{0}(q) \delta\left(q+q^{\prime}\right) e^{\omega(q)\left(t+t^{\prime}\right)},
$$

where we have considered the symmetry $\omega(-q)=\omega(q)$. The two subsequent terms in (28) do not contribute,

$$
C_{2}=C_{3}=0,
$$

because the the initial condition is a random variable independent of the Brownian process, $W$. For the term $C_{4}$, given in (32), we note that since a Brownian evolution up to a certain time is independent of later increments, only the common interval $\left[0, t \wedge t^{\prime}\right]$ contributes to the correlation of the product of the integrals. Besides, due to Eq. (27), only the terms with $q^{\prime}=-q$ have nonzero correlation. Thus, we obtain

$$
\begin{aligned}
C_{4}= & -2 \sigma \tilde{h}_{0}^{3} q^{2} 2 \pi \delta\left(q+q^{\prime}\right) E \\
& \times\left[\int_{0}^{t \wedge t^{\prime}} e^{\omega(q)(t-s)} d \widehat{W}(s) \int_{0}^{t \wedge t^{\prime}} e^{\omega(q)\left(t^{\prime}-s^{\prime}\right)} d \widehat{W}\left(s^{\prime}\right)\right]
\end{aligned}
$$

$$
=-2 \sigma \tilde{h}_{0}^{3} q^{2} \delta\left(q+q^{\prime}\right) \widehat{F}(q) \int_{0}^{t \wedge t^{\prime}} e^{\omega(q)(t-s)} e^{\omega(q)\left(t^{\prime}-s\right)} d s .
$$

The last line above is a consequence of a well-known property of Ito's integral $[23,24]$. Performing the integral and using $t+t^{\prime}-2\left(t \wedge t^{\prime}\right)=\left|t-t^{\prime}\right|$, we have

$$
C_{4}=\sigma \tilde{h}_{0}^{3} 2 \pi \delta\left(q+q^{\prime}\right) \frac{q^{2} \widehat{F}(q)}{\omega(q)}\left[e^{\omega(q)\left(t+t^{\prime}\right)}-e^{\omega(q)\left|t-t^{\prime}\right|}\right] .
$$

Finally, by replacing Eqs. (34), (35), and (37) in Eq. (28), we obtain

$$
\left\langle\delta \widehat{h}(q, t) \delta \widehat{h}^{\prime}\left(q^{\prime}, t^{\prime}\right)\right\rangle=2 \pi \delta\left(q+q^{\prime}\right) S\left(q ; t, t^{\prime}\right),
$$

where

$$
\begin{aligned}
S\left(q ; t, t^{\prime}\right)= & \widehat{F}_{0}(q) e^{w(q)\left(t+t^{\prime}\right)}+\sigma \tilde{h}_{0}^{3} \frac{q^{2} \widehat{F}(q)}{\omega(q)} \\
& \times\left[e^{\omega(q)\left(t+t^{\prime}\right)}-e^{\omega(q)\left|t-t^{\prime}\right|}\right] .
\end{aligned}
$$

For the case of noncorrelated noise, we have $\widehat{F}(q)=1$, in which case we obtain the relation given in Ref. [8].

The first term of Eq. (39) corresponds to the spectra predicted by the deterministic model $(\sigma=0)$. In the following we shall compare the evolution of films with $(\sigma>0)$ and without the stochastic term. In the later case, the film has to be perturbed at $t=0$, otherwise no evolution is triggered. We shall assume that the originally flat free surface of the film is slightly modified by a perturbation adding no flow at the boundaries of a space domain chosen to be the interval $[0, L]$. Such a perturbation admits a sine Fourier transform

$$
\delta h(x, 0)=\sum_{k=1}^{N} B_{k} \sin (2 \pi x k / L)
$$

whence we obtain $F_{0}(q)=\widehat{\delta h}(q, 0)$. The (small) amplitudes $B_{k}$ are chosen as random numbers with $\left|B_{k}\right|<B_{\max }=10^{-3} \tilde{h}_{0}$.

As a typical case, in the following calculations we choose a film with $h_{*}=0.1$ and $\theta=30^{\circ}$, which yields [22] $q_{m}=0.151, q_{c}=0.213$, and $\omega_{m}=5.1910^{-4}$. Even if only a few terms of Eq. (40) are expected to be relevant, we take $N=50$. The quantities $\lambda_{m}=2 \pi / q_{m}=41.6$ and $\tau_{m}=\left(1 / \omega_{m}\right) \ln \left[\left(\tilde{h}_{0}-h_{*}\right) / B_{\max }\right]=13113.5$ give a rough idea of the spatial extension and time duration of the film breakup process. We find that $L=500 \approx 12 \lambda_{m}$ is large enough to produce results that are independent of the domain size. The consequences on the stochastic process of using a correlated noise on a finite domain is analyzed in the next section.

\section{B. Correlated stochastic noise in a finite domain}

Here we will assume that the correlation function $F$ in Eq. (5) is $L$ periodic. Note that it is a matter of convention whether an $L$-periodic domain is considered a finite torus or an infinite domain obtained by subsequently pasting copies of the fundamental $L$ cell and considering only solutions invariant under $L$ translations. We prefer the latter visualization. 
In this case the stochastic process $\Theta(x, t)$ can be expanded [19] in terms of functions of separated variables in the form

$$
\Theta(x, t)=\frac{\partial W(x, t)}{\partial t}=\sum_{k=-\infty}^{+\infty} \chi_{k} \dot{c}_{k}(t) g_{k}(x),
$$

where the coefficients $\dot{c}_{k}$ correspond to white-noise processes obtained as (weak) time derivatives of mutually independent Brownian motions $c_{k}$, and the functions $g_{k}$ form the complete set of orthonormal eigenfunctions

$$
g_{k}(x)= \begin{cases}\sqrt{\frac{2}{L}} \cos (2 \pi k x / L), & k>0 \\ \sqrt{\frac{1}{L}}, & k=0 \\ \sqrt{\frac{2}{L}} \sin (2 \pi k x / L x), & k<0\end{cases}
$$

of the Hilbert-Schmidt operator $\mathcal{Q}$ defined by

$$
\mathcal{Q} f(x)=\int_{-L / 2}^{L / 2} F\left(x-x^{\prime}\right) f\left(x^{\prime}\right) d x^{\prime} .
$$

The constants $\chi_{k}$ are the eigenvalues corresponding to each $g_{k}$ :

$$
\mathcal{Q} g_{k}(x)=\chi_{k} g_{k}(x) \text {. }
$$

In fact,

$$
\chi_{k}=\int_{-L / 2}^{L / 2} F(u) e^{-i 2 \pi k u / L} d u .
$$

Equations (43)-(45) are a consequence of the following simple calculation. If $G_{k}(x)=e^{-i q_{k} x}$ with $q_{k}=2 \pi k / L$, then

$$
\begin{aligned}
\mathcal{Q} G_{k}(x) & =\int_{-L / 2}^{L / 2} F\left(x-x^{\prime}\right) e^{-i q_{k} x^{\prime}} d x^{\prime} \\
& =e^{-i q_{k} x} \int_{-L / 2-x}^{L / 2-x} F(u) e^{-i q_{k} u} d u=\chi_{k} G_{k}(x) .
\end{aligned}
$$

The second equality uses the symmetry property $F(u)=$ $F(-u)$ and the last one the fact that, by $L$ periodicity, the $x$ dependence at the limits of integration can be omitted. Note that Eq. (45) is the finite-size domain version of Eq. (26) for a discrete spectrum, so the correlated noise effect is embedded in the discrete spectrum of the Hilbert-Schmidt operator $\mathcal{Q}$.

We choose the particular correlation function [19]

$$
F\left(u, \ell_{c}\right)= \begin{cases}Z^{-1} \exp \left[-\frac{1}{2}\left(\frac{L}{\ell_{c}} \sin \left(\frac{\pi u}{L}\right)\right)^{2}\right], & \ell_{c}>0 \\ \delta(u), & \ell_{c}=0,\end{cases}
$$

where $\ell_{c}$ is the correlation length and $Z$ is such that $\int_{0}^{L} F\left(u, \ell_{c}\right) d u=1$. This function represents the equilibrium distribution of the height of an oscillating surface subjected to a (linear) surface tension $L / \ell_{c}$. For this correlation, the eigenvalues in Eq. (45) (see Appendix) are

$$
\widehat{F}\left(q_{k}\right)=\chi_{k}=\frac{I_{k}(\alpha)}{I_{0}(\alpha)},
$$

where

$$
\alpha=\left(\frac{L}{2 \ell_{c}}\right)^{2}=: \beta^{2}
$$

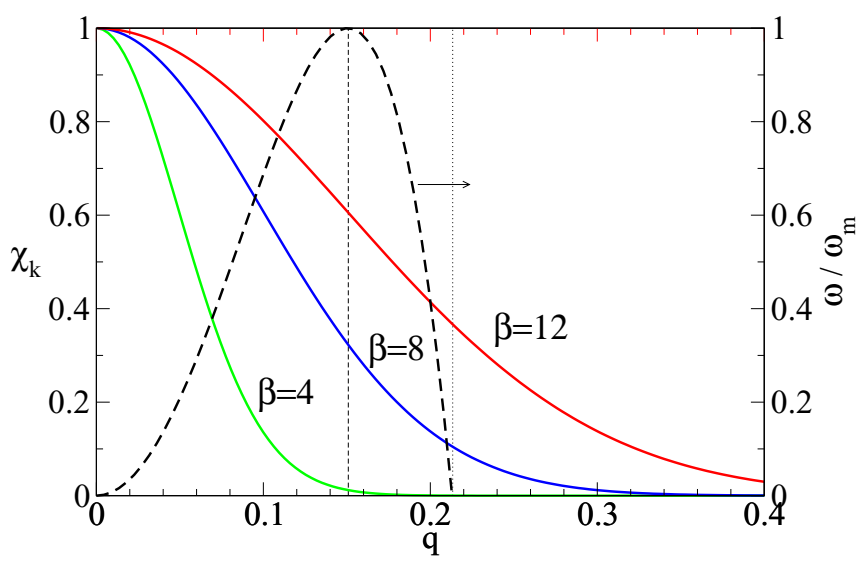

FIG. 1. Linear spectrum of eigenvalues for several values of $\beta$ obtained from Eqs. (48) and (49). The vertical lines indicate the values of $q_{c}$ and $q_{m}$, while the dashed curve corresponds to the deterministic dispersion relation, $\omega(q)$, given by Eq. (20). Here we take $L=500$ to evaluate $k=q L /(2 \pi)$.

We show in Fig. 1 this eigenvalue spectrum for several values of $\beta=L /\left(2 \ell_{c}\right)$. Note that for $\beta \rightarrow \infty$ (i.e., $\ell_{c} \rightarrow 0$ ), we have $\chi_{k} \rightarrow 1$ for all $k$, which leads to the limiting case of white (uncorrelated) noise. For decreasing $\beta$ (larger $\ell_{c}$ 's) the width of the spectrum curve diminishes monotonically. The effect of the correlation region (i.e., not negligible values of $\chi_{k}$ ) on the film instability can be put in evidence by comparing it with the dispersion relation $\omega(q)$ as given by the deterministic LSA, Eq. (20) (see dashed line in Fig. 1). For $\beta \gtrsim 8$, all modes (stable and unstable ones) are affected by the noise with increasing effect on stable ones as $\beta$ increases. On the other hand, for $\beta \lesssim 8$ only unstable modes are affected by the thermal noise. Note that this limiting value is related to the value of $\ell_{c}$, so both the periodicity of the problem, $L$, and the wavelength of maximum growth, $\lambda_{m}$, play a role in the determination of these regions.

The actual effect of $\ell_{c}$ on the evolution of the instability is clearly observed in the power spectrum of the perturbation, $S(q, t)$, as predicted by the linear stability analysis in Sec. III. Figure 2 shows $S$ versus $q$ at $t=200$ and $t=2000$ as given by Eqs. (39) $\left(t=t^{\prime}\right)$ and (40). As expected from the analysis of Fig. 1, the inclusion of stochastic noise increases the amplitude of the modes with $q>q_{c}$ (dotted vertical line) which are otherwise stable in the deterministic case. This effect increases with $\beta$, as the noise becomes closer to a white noise $\left(\ell_{c} \rightarrow 0\right)$.

In Fig. 3 we show the time evolution of the wave number of the maximum of the spectra, $q_{\max }(t)$, for different values of $\beta$. Note that for $\beta \lesssim 9$, we find $q_{\max }<q_{m}$, while we have $q_{\max }>q_{m}$ for larger $\beta$. Therefore, $q_{\max }$ approaches $q_{m}$ from below for $\beta \lesssim 9$ and from above for larger $\beta$.

In order to understand this behavior, we first analyze what determines the value of $q_{\max 0}=q_{\max }(t=0)$. To do so, we consider the derivative of Eq. (39) with respect to $q$ for small times (i.e., $\omega t \ll 1$ ) and find that $q_{\max 0}$ is given simply by the maximum of $q^{2} \chi(q)$. By using the approximate expression

$$
I_{k}(\alpha) \approx \frac{e^{\alpha}}{\sqrt{2 \pi \alpha}}\left(1-\frac{4 k^{2}-1}{8 \alpha}+\ldots\right)
$$




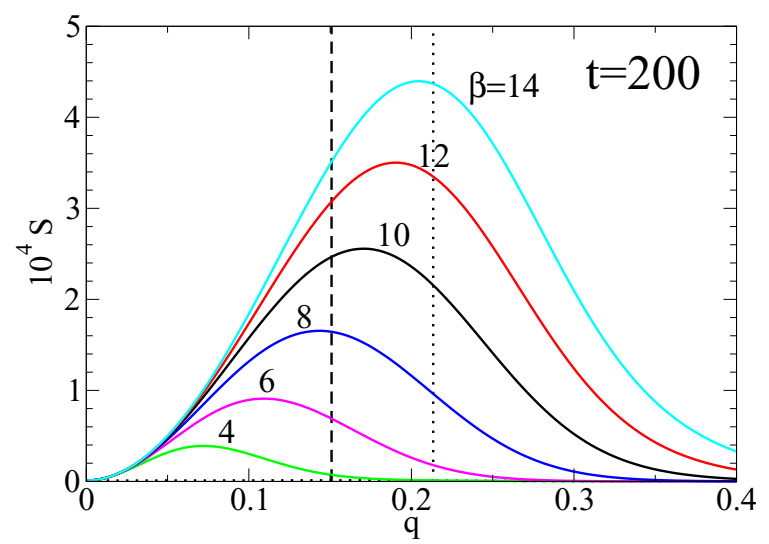

(a) $t=200$

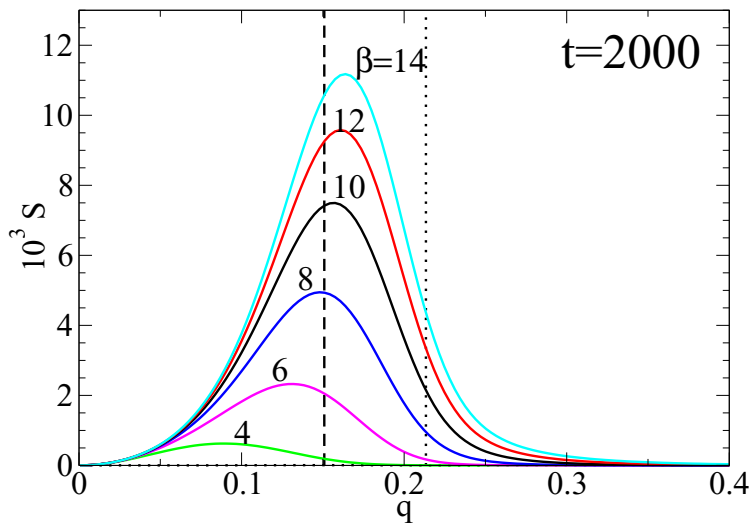

(b) $t=2000$

FIG. 2. Power spectrum at two different times for $\sigma=5 \times 10^{-5}$, and several values of $\beta$ as given by the linear prediction in Eq. (39) and the initial perturbation in Eq. (40). The vertical dashed and dotted lines correspond to the wave number of maximum growth rate $\left(q_{m}=2 \pi / \lambda_{m}\right)$ and marginal stability $\left(q_{c}=2 \pi / \lambda_{c}\right)$, respectively.

for large $\alpha$, we find

$$
q_{\max 0}=2 \sqrt{2} \pi \frac{\beta}{L} .
$$

Thus, the condition $q_{\max 0}=q_{m}$ yields $\beta_{c}=8.492$ for $L=$ 500, as shown in Fig. 3. Interestingly, this expression points out that this condition occurs when $\ell_{c}=\lambda_{c}$ for any value of $L$. Therefore, the maximum of the spectrum $S(q, t), q_{\max }(t)$, remains below $q_{m}$ when the correlation length, $\ell_{c}$, is less than the critical wave number and vice versa. In the white-noise case, this maximum is always above $q_{m}$, and $q_{\max 0}=\infty$.

\section{NUMERICAL IMPLEMENTATION IN A FINITE DOMAIN}

In order to understand the nonlinear effects in the film instability, we perform numerical simulations of the evolution of the film governed by the nonlinear Eq. (14). The calculations are carried out in a computational domain defined by $0 \leqslant x \leqslant$ $L$, which is divided into cells of size $\Delta x$. Typically, we use $\Delta x=0.1=h_{*}$, which assures convergence of the numerical

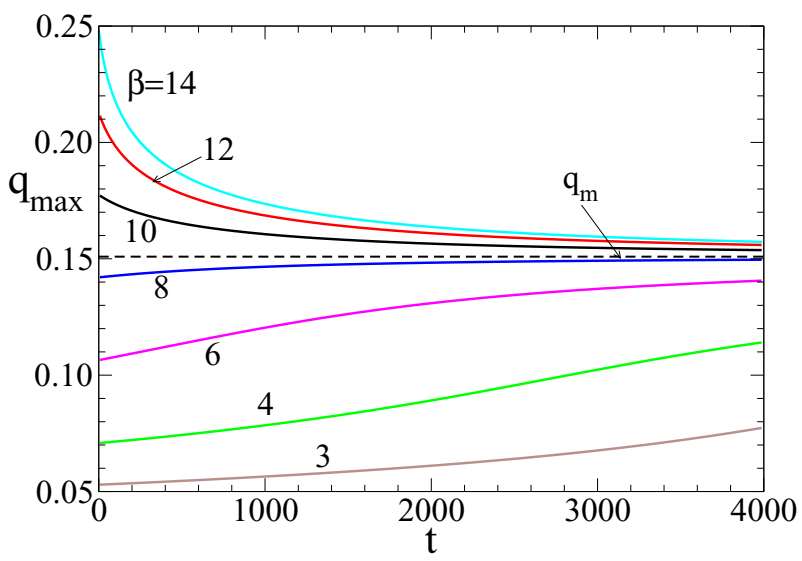

FIG. 3. Time evolution of the wave number of the maximum of the spectra, $q_{\max }$, for different values of $\beta$. Note that all the stochastic timelines of $q_{\max }$ asymptote the deterministic value $q_{m}$ for long times. scheme [26], and by setting $L=500$ as mentioned above, we have 5000 cells.

Equation (14) is discretized in space using a central finite difference scheme with periodic boundary conditions. Time discretization is performed using implicit Crank-Nicolson scheme with relaxation factor equal to $1 / 2$. Thus, the time evolution of the stochastic term is performed according to Stratonovich rules. In fact, symmetry considerations imply that Ito and Stratonovich calculus are equivalent for the integration of Eq. (14) [19]. We note that all the results presented in this paper are fully converged, as verified by grid refinement; more details about numerical issues can be found in Ref. [27]. Note that the minimum possible value of the correlation length is $\ell_{c}=\Delta x(=0.1$ in our case $)$, since the discretized equations cannot distinguish any correlation below this length scale. Thus, the limiting case of white noise, which corresponds to $\ell_{c}=0$ (i.e., $\beta=\infty$ and $\chi_{k}=1$ ), cannot be calculated numerically with accuracy, and, consequently, this limit is studied by observing the trends as $\beta$ increases.

To represent the time-Wiener processes in the framework of Ito calculus using a discrete form, we replace $\dot{c}_{k}\left(t_{n}\right)$ at a time step $t_{n}$ by the forward difference quotient

$$
\dot{c}_{k}\left(t_{n}\right) \approx \frac{\Delta c}{\Delta t_{n}}=\frac{c_{k}\left(t_{n+1}\right)-c_{k}\left(t_{n}\right)}{t_{n+1}-t_{n}} .
$$

The difference $\Delta c$ is normal distributed and the variance is given by the time increment $\Delta t_{n}$. Thus, we approximate Eq. (52) by

$$
\frac{\Delta c}{\Delta t_{n}}=\frac{\mathcal{N}_{k}^{n}}{\sqrt{\Delta t_{n}}},
$$

where $\mathcal{N}_{k}^{n}$ is a computer-generated random number which is approximately $N(0,1)$ distributed, i.e., its histogram is close to a Gaussian with mean zero and unity standard deviation (we used the GASDEV routine from Ref. [28]). Altogether, the space-time discrete noise term, Eq. (41), is given by

$$
\Theta(x, t)=\frac{1}{\sqrt{\Delta t_{n}}} \sum_{k=-\frac{N-1}{2}}^{\frac{N-1}{2}} \chi_{k} \mathcal{N}_{k}^{n} g_{k}(x),
$$



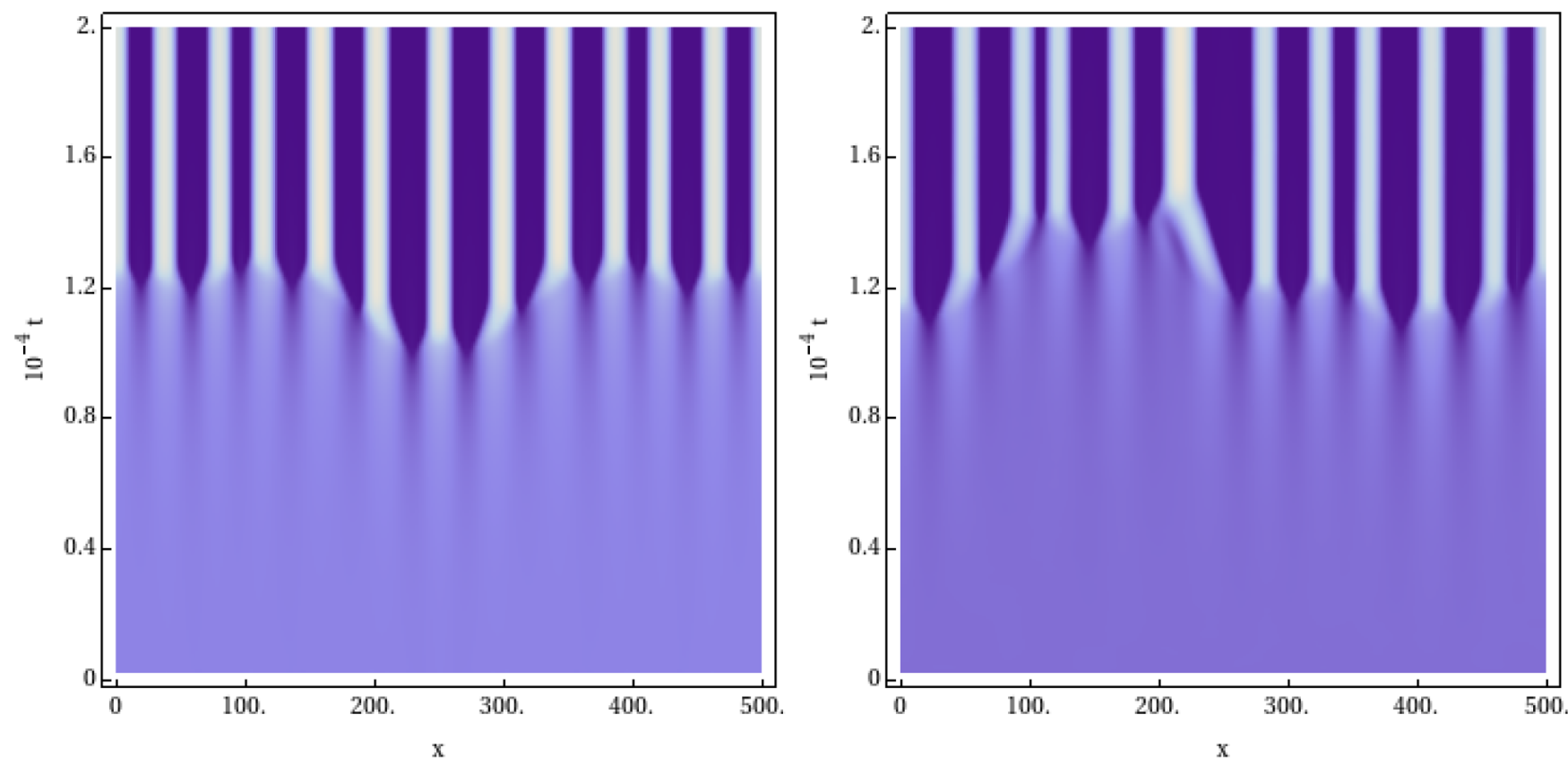

(a) $\sigma=0$

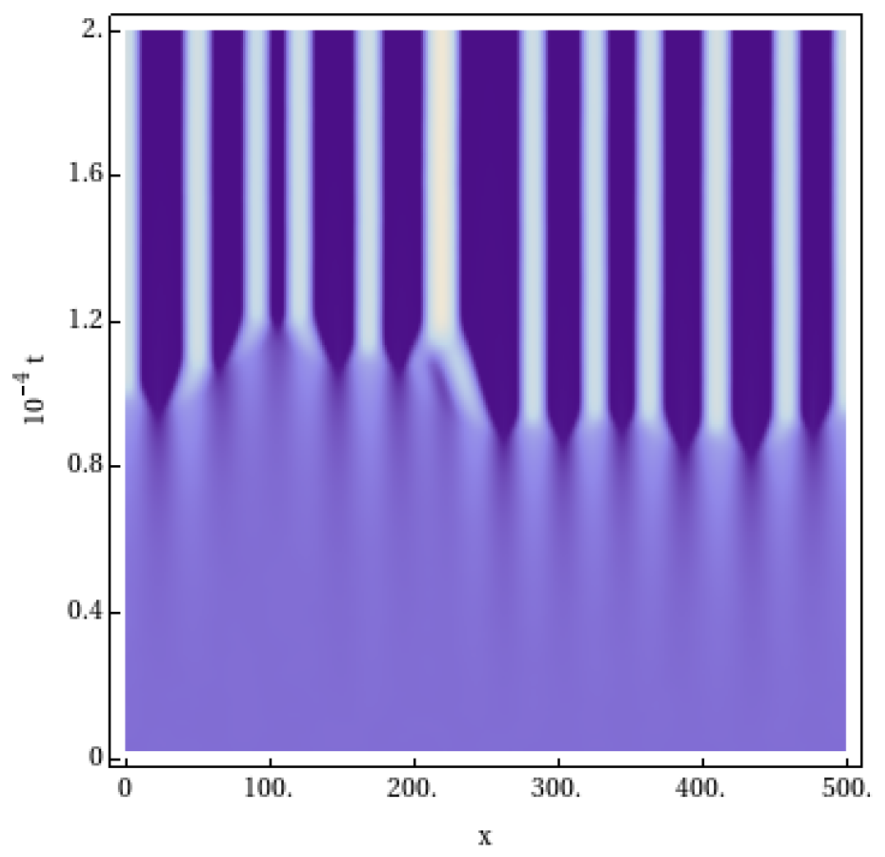

(c) $\sigma>0, \beta=6$

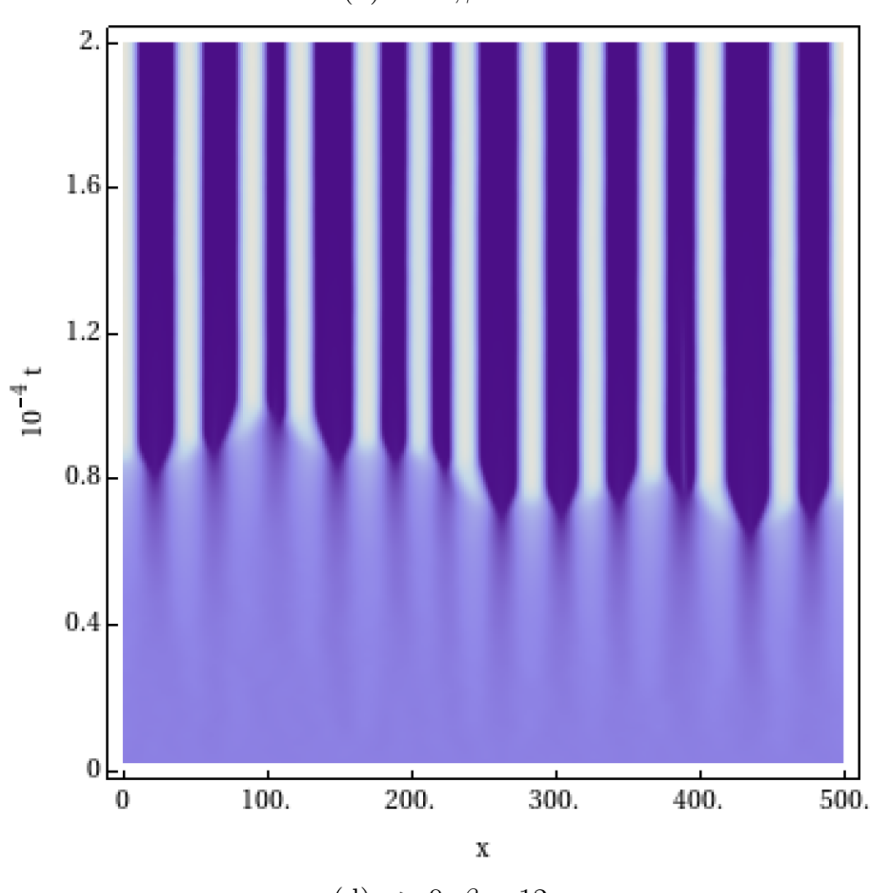

FIG. 4. Space-time plot of $h(x, t)$ for (a) the deterministic case $(\sigma=0)$ and stochastic cases $\left(\sigma=5 \times 10^{-5}\right)$ with spatially correlated noise (b) $\beta=4$, (c) $\beta=6$, and (d) $\beta=12$ (very close to white noise). Darker regions correspond to smaller thicknesses.

where $\chi_{k}$ is given by Eq. (48) and $g_{k}(x)$ by Eq. (42). Thus, Eq. (54) is used to calculate the noise term in Eq. (14).

Each realization of the stochastic process requires a given seed for $\mathcal{N}$. Then some of the numerical results presented below correspond to a single realization and others to the average of 60 realizations (different seeds). A typical example of the evolution of a film for a single realization (i.e., a given seed) is shown in the space-time plots shown in Fig. 4 for $\sigma=0$ and increasing values of $\beta$ for $\sigma=5 \times 10^{-5}$. Here darker regions correspond to smaller thicknesses. Even for these single realizations, some effects of the noise can be observed. For instance, we notice that an important effect is to decrease the duration of the breakup process with respect to the deterministic case $(\sigma=0)$. Note also that the final number of drops is reduced when spatially correlated noise is important, i.e., $\beta<\beta_{c}(=8.492$ in our case $)$. This reduction is due to merging of thickness peaks as the instability evolves, and this effect is more frequent as $\sigma$ increases (not shown for brevity). The final pattern for $\beta>\beta_{c}$ is very similar to that shown 


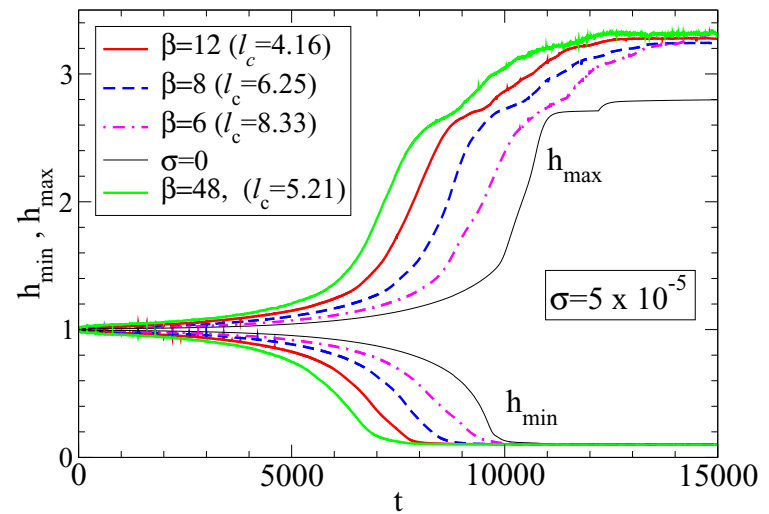

(a)

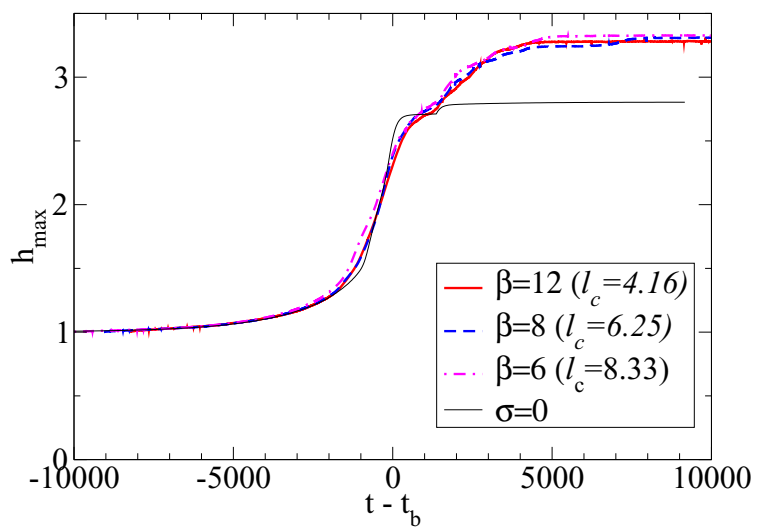

(b)

FIG. 5. (a) Average maximum, $h_{\max }$, and average minimum, $h_{\min }$, of film thickness versus time for several values of $\ell_{c}$ and $\sigma=5 \times 10^{-5}$ over 60 realizations. (b) Average maximum thickness, $h_{\max }$, versus the shifted time $t-t_{b}$, where $t_{b}=8250,9062$, and 10020 for $\beta=12,8$, and $6\left(\ell_{c}=4.16,6.25\right.$, and 8.33.)

in Fig. 4(d) for $\beta=12$, so this case is representative of the white-noise limit.

In order to study how the correlated noise affects the time evolution of the instability we first concentrate on the time it takesfor the first rupture of the film to appear. By first rupture time, we mean the moment when the film first reaches its possible smallest value, which is $h_{*}$. Figure 5(a) shows the time evolution of the average of the minimum of $h(x, t)$, namely $h_{\text {min }}(t)$. Clearly, as $\beta$ decreases the breakup time, $t_{b}$, increases, such that as $\beta \rightarrow 0\left(\ell_{c} \rightarrow \infty\right) t_{b}$ tends to the value given by the case without noise $(\sigma=0)$, which has the largest time. On the contrary, $t_{b}$ decreases as $\beta \rightarrow 0$, and the noise becomes less correlated and tends to white noise in space. For $\sigma>0$, this time decreases for increasing $\sigma$.

A parameter of interest for the drop formation problem after the first breakup is the evolution of the maximum thickness as the final static configuration is reached. In Fig. 5(a) we show the average of $h_{\max }(t)$ for different values of $\beta$. We also plot $h_{\min }(t)$ for reference and define the corresponding breakup times, $t_{b}$, as $h\left(t_{b}\right)=1.05 h_{*}=0.0105$. Figure 5(b) shows that in fact the evolution of $h_{\text {max }}(t)$ is very weakly dependent on $\beta$ (i.e., $\ell_{c}$ ), since the curves $h_{\max }$ versus $t-t_{b}$ are practically superimposed. This result implies that the noise does not have any effect on the drop formation process after the breakup of the film, that is, during the dewetting stage following the pinch off.

Now we aim to study the effects of the correlation length in both linear (early) and nonlinear (late) stages of the instability. To do so, we calculate the Fourier spectra of the thickness profiles for different times. In Fig. 6 we show the evolution of the spectra with $\beta=12\left(\ell_{c}=4.16\right)$ for both early and late times. All spectra correspond to an average over 60 realizations, and no adjusting parameter has been used (the scales for $S$ differ from those used in previous sections because a different normalization was employed in the Fourier transform of the numerical results). For early times, the agreement between numerics and the linear stability prediction, Eq. (39), is very good. For larger times, the peaks of both spectra approach $q_{m}$ though the numerics show higher and a bit wider spectra than those predicted by LSA. A similar situation is observed for smaller values of $\beta$ as shown in Fig. 7.
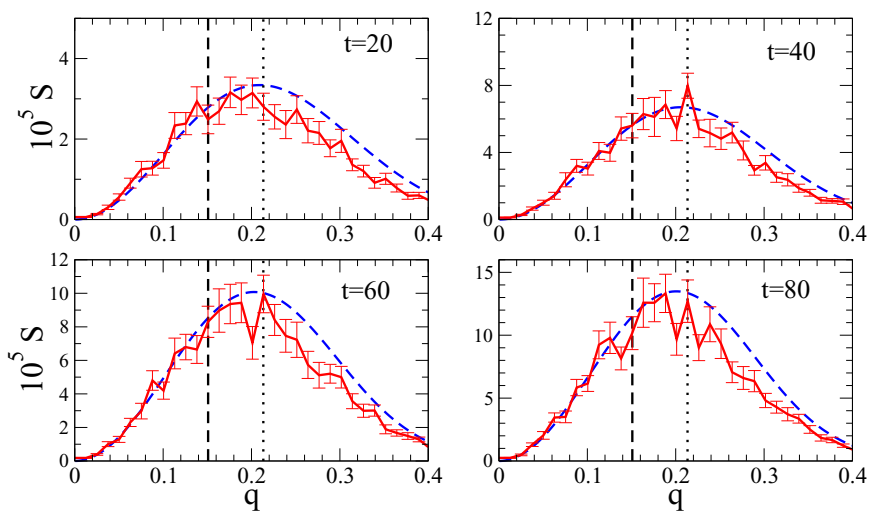

(a) $\beta=12$, early times
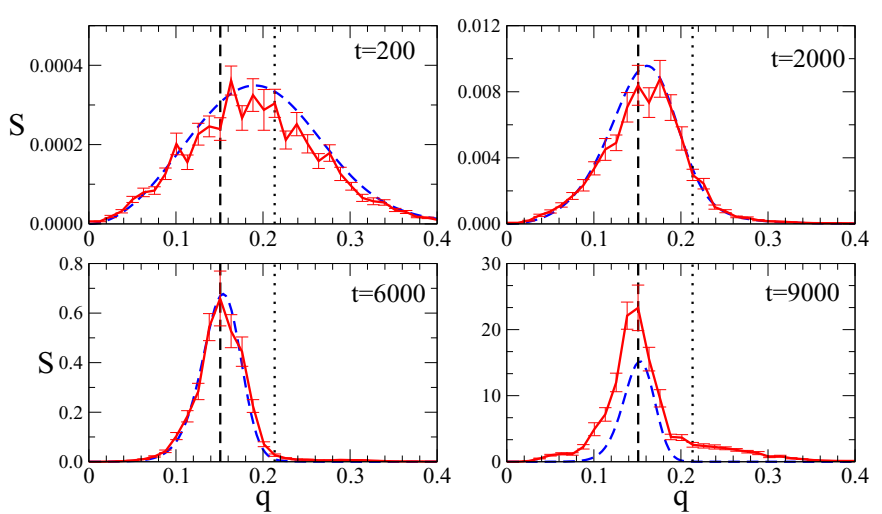

(b) $\beta=12$, late times

FIG. 6. Numerical power spectra, $S(q, t)$ (solid lines), for (a) early and (b) late times for $\sigma=5 \times 10^{-5}$ and $\beta=12\left(\ell_{c}=4.16\right)$ averaged for 60 realizations of the problem defined in Fig. 4(d). The dashed lines are the corresponding predictions of the LSA, and the error bars show the standard deviation of the mean. The vertical dashed line corresponds to the wave number of maximum growth in the deterministic case, $q_{m}=0.151$, while the dotted one corresponds to the marginal value, $q_{c}=0.215$. 


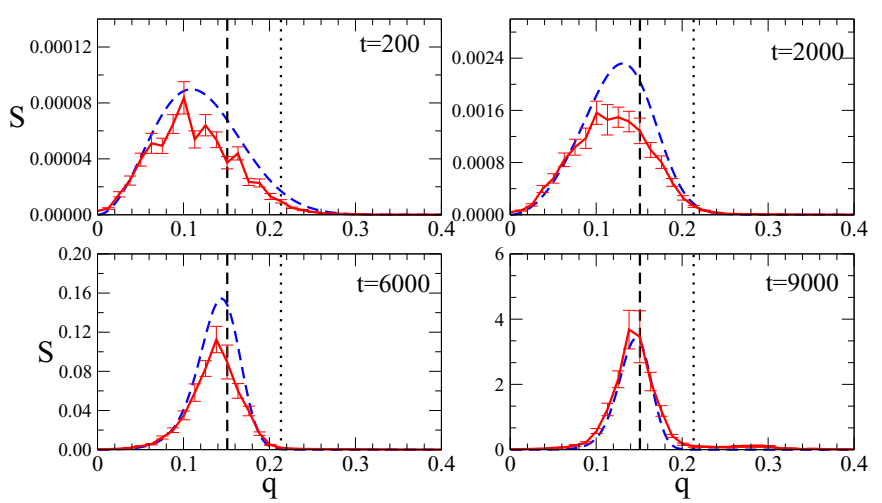

(a) $\beta=6$
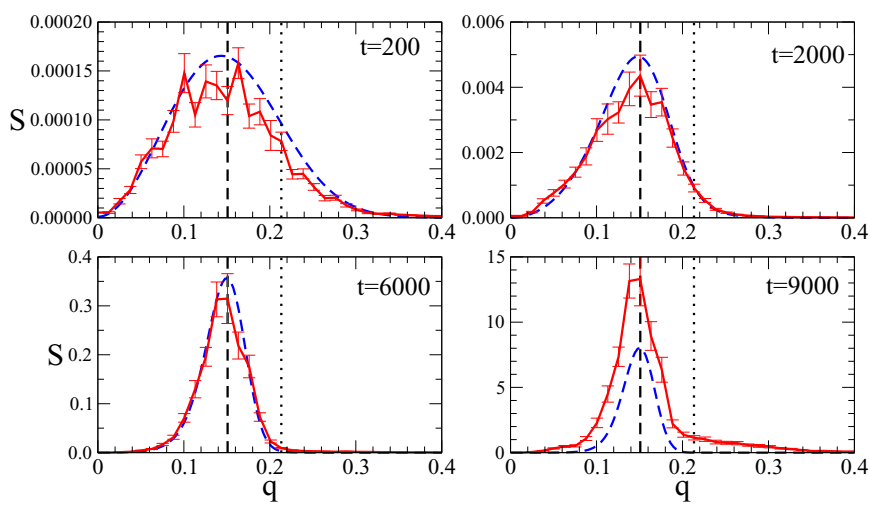

(b) $\beta=8$

FIG. 7. Numerical power spectra, $S(q, t)$ (solid lines), for $\sigma=5 \times 10^{-5}$ and (a) $\beta=6\left(\ell_{c}=8.33\right.$ ) and (b) $\beta=8\left(\ell_{c}=6.25\right)$ averaged for 60 realizations. The thick red lines correspond to moving average curves of the gray lines (raw data). The dashed lines are the corresponding predictions of the LSA, and the error bars show the standard deviation of the mean. The vertical dashed line corresponds to the wave number of maximum growth in the deterministic case, $q_{m}=0.151$, while the dotted one corresponds to the marginal value, $q_{c}=0.215$.

\section{COMPARISON WITH EXPERIMENTS}

Previous comparisons between experiments and stochastic models have studied the instability of polymeric films on silicon oxide substrates $[15,16]$. However, these comparisons were made without considering spatial correlation, i.e., assuming both spatial and temporal white noise. Also, they mainly employed the integration of the spectra $S(q)$ for all possible values of $q$ and derived quantities from it. Here, instead, we apply the theoretical model described above to experimental results for unstable liquid metal films to evaluate the importance of spatial correlations when considering stochastic instabilities. In order to do this, we do not restrict ourselves to some integrals of the spectra but employ their complete profiles as a function of the wave number, $q$.

Our experimental data correspond to copper thin films of a few nanometers thick that are melted by the illumination with pulses of an excimer laser that last some tens of nanoseconds. During these pulses, the metal is in a liquid state, and thus the present hydrodynamic model can be applied. In this configuration, the liquid lifetime of the melted copper is related with the local temperature of the film, i.e., with the spatial distribution of the laser intensity, which spans in a radially symmetric Gaussian profile. After the pulse, the metal solidifies, leaving a distinct pattern of holes, drops, and/or ridges depending on how long the metal has been in the liquid state. More information about this setup configuration and details on the technique can be found elsewhere [29-33].

Since the outer regions of the laser spot have shorter liquid lifetimes, one can associate these regions with earlier times of the evolution and, consequently, central regions with later times. Since the laser spot is relatively large, the SEM images of these experiments have the advantage of offering more spatial information than other setups [15]. Nevertheless, they have the drawback that the times corresponding to every stage of the evolution are unknown, even if it is possible to order the time sequence in connection with the distance of the image respect to the center of the laser spot [18]. The goal of the following comparison is to show that the experimental observations represented by the spectra require not only a stochastic temporal evolution but also some spatial correlation in the thermal noise in order to reproduce the full results.

In particular, we will concentrate here on the data reported in Ref. [18], where the SEM images of the evolving melted metal were analyzed by using bidimensional (2D) discrete Fourier transform (DFT). Since the 2D spectra turned out to be radially symmetric in the wave-number space, $\left(q_{x}, q_{y}\right)$, the results in Fig. 5 of Ref. [18] were reported as amplitudes $A_{\text {2D }}$ versus $k=\left(q_{x}^{2}+q_{y}^{2}\right)^{1 / 2}$. Therefore, the corresponding $1 \mathrm{D}$ correlation is obtained as $S=k A_{2 \mathrm{D}}^{2}$ (see the symbols in Fig. 8). The symbols for both small $k$ and amplitudes $(S<0.15)$ are an artifact of the finite length of the sample in the Fourier calculation. Note that this effect does not change in time. Its importance decreases when the evolution of the instability yields a peak with a characteristic length and, as a consequence, this part of the spectrum close to $q=0$ becomes less relevant. Therefore, the fittings can be done without taking into account these data for very small $k$, since the main peaks are not affected in any meaningful way by them.

The parameters for liquid copper are $\gamma=1.304 \mathrm{~N} / \mathrm{m}$ and $\mu=4.38 \mathrm{mPas}$. Assuming $T=1500 \mathrm{~K}$ as a typical temperature of the film with thickness $h_{0}=8 \mathrm{~nm}$, we have $\sigma=2.48 \times 10^{-4}$ and $t_{0}=0.08 \mathrm{~ns}$. Regarding the intermolecular interaction with $\mathrm{SiO}_{2}$ we use $h_{*}=0.1 \mathrm{~nm}$ and $A=$ $2.58 \times 10^{-18} \mathrm{~J}$ (as suggested in Ref. [18]). Thus, we have $q_{c}=63.4 \mu \mathrm{m}^{-1}$ and $q_{m}=44.8 \mu \mathrm{m}^{-1}$ (dotted and dashed lines in Fig. 8).

In order to perform the comparison of the experimental and theoretical spectra [see Eq. (39)] we choose a constant value for the unknown $\widehat{F}_{0}(q)$, namely $\widehat{F}_{0}(q)=2 \times 10^{-4}$, and use the same normalization factor for the DFT as in Ref. [18]. Thus, we are left only with $t$ and $\beta$ as adjustable parameters. The fitting values for the spectra in Fig. 8 are given in Table I. The low local maximum for $k \approx 100 \mu \mathrm{m}^{-1}$ is related to the size of the drops, which is smaller than the distance between them [18].

Interestingly, we find not only increasing values of time as one moves from inner to outer regions (as expected) but also a decrease of the corresponding values of $\beta$ required for the fitting. This implies that the stochastic noise somehow differs at the sampled regions which, in turn, correspond to distinct liquid lifetimes. However, the relatively large values of 


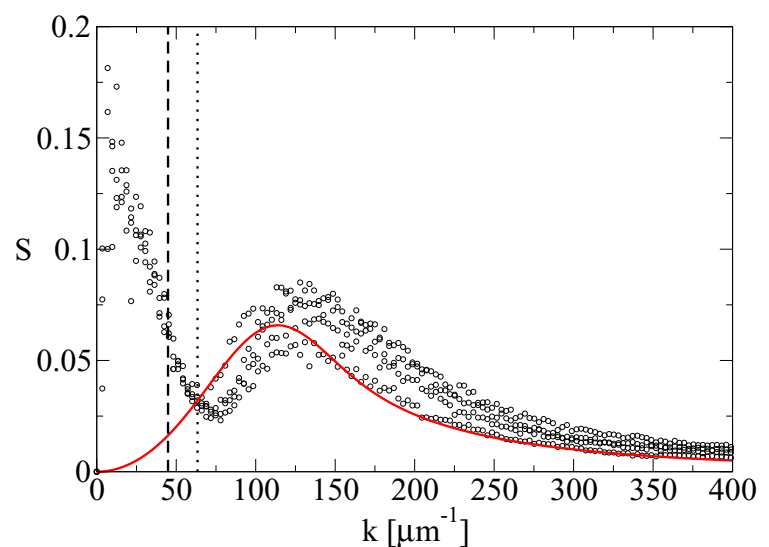

(a)

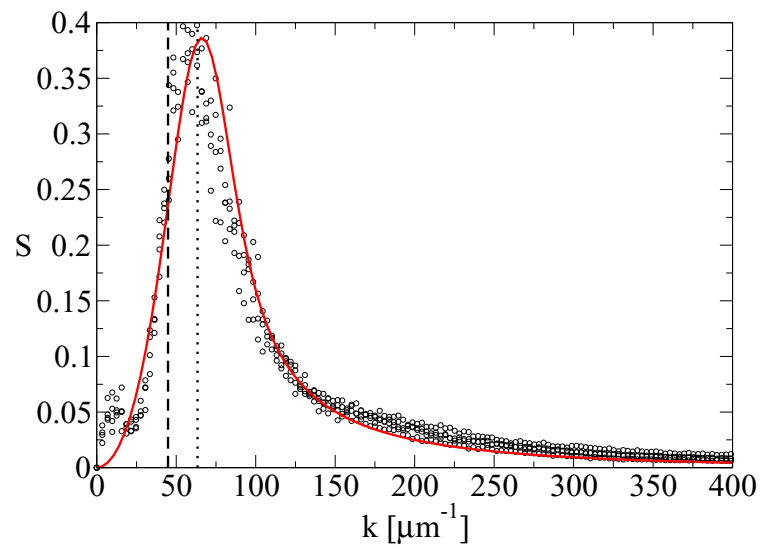

(c)

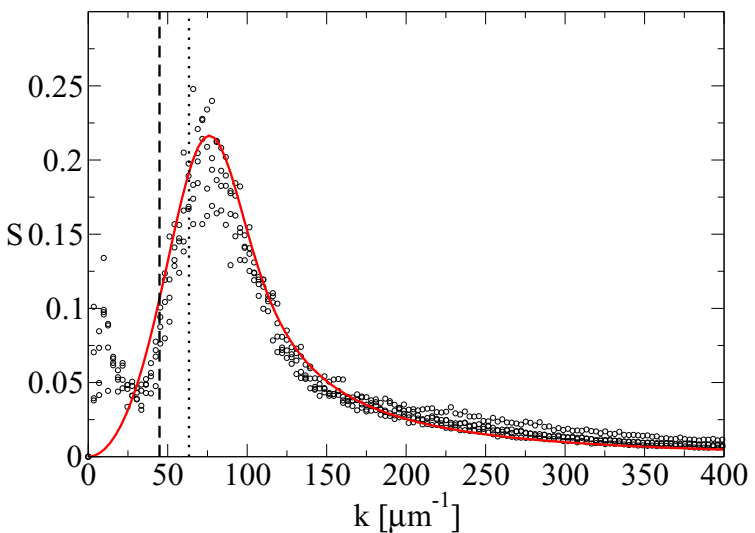

(b)

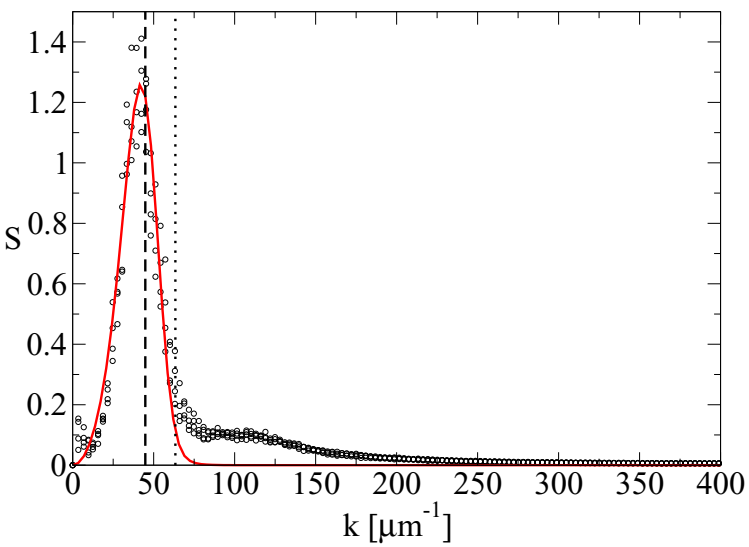

(d)

FIG. 8. Experimental power spectra, $A_{1 \mathrm{D}}(k, t)$, (symbols) from Fig. 5 of Ref. [18], and theoretical spectra (solid lines) obtained with the present stochastic model with spatial correlation. The experimental spectra are organized in decreasing order of their distance to the center of the laser spot.

$\beta$ for the first three images suggest that the noise is practically white at the beginning and that spatial correlation becomes important only for larger times when $\beta$ decreases significantly. In general, it is then expected that the spectrum for earlier times (i.e., near the outer borders of the laser spot) correspond to a quasi-white noise, but the noise becomes more and more spatially correlated as one goes to the center of the spot (i.e., as the liquid lifetimes increase). In fact, the correlation length, $\ell_{c}$, can be estimated considering the value of $\beta$ and the length of the image, which can be assumed as the periodicity length, $L$. For the images corresponding to Fig. 8 we have $L=2.13 \mu \mathrm{m}$, so we obtain $\ell_{c}=L /(2 \beta)$ as shown in Table I. Moreover, note that $\ell_{c}$ finally approaches $\lambda_{m}(=144 \mathrm{~nm})$, which is also very close to $\lambda_{m}^{\exp }(=165 \mathrm{~nm})$. Thus, $\ell_{c}$ turns out to be very close to the average distance between drops.

TABLE I. Best fit values from the comparison of the stochastic model with spatial correlation with experimental spectra of unstable liquid metal films. Here we have $\lambda_{m}=144 \mathrm{~nm}$.

\begin{tabular}{lcccccc}
\hline \hline Fig. 8 & $t(\mathrm{~ns})$ & $\beta$ & $\ell_{c}(\mathrm{~nm})$ & $\lambda_{m} / \ell_{c}$ & $\lambda_{m}^{\exp }(\mathrm{nm})$ & $\lambda_{m}^{\exp } / \ell_{c}$ \\
\hline (a) & 0.08 & 175 & 6.1 & 22.9 & 62.8 & 10.3 \\
(b) & 0.48 & 160 & 6.6 & 21.0 & 99.7 & 14.9 \\
(c) & 0.97 & 140 & 7.6 & 18.4 & 125.6 & 16.5 \\
(d) & 6.21 & 7.4 & 144.1 & 0.97 & 165.3 & 1.15 \\
\hline \hline
\end{tabular}

\section{SUMMARY AND CONCLUSIONS}

In this work we have considered the effect of correlated thermal noise on the instability of a liquid thin film under the action of viscous, capillary, and intermolecular forces by adding a stochastic term in the lubrication approximation equation for the film thickness. This term depends on the noise amplitude that is spatially self-correlated within a characteristic microscopic distance, $\ell_{c}$. The LSA of the resulting equation shows that this yields a new factor in the stochastic part of the instability spectrum [or dispersion relation, $\omega(q)$ ], which is given by the Fourier transform of the correlation function that can be expressed in terms of the eigenvalues of the Hilbert operator associated with it.

In order to observe the nonlinear effects on the evolution of the instability, we also perform numerical simulations of the full lubrication equation using different seeds to generate the random sequence of amplitudes for the stochastic term (so a realization corresponds to each seed) and average the resulting power spectra to obtain a representative spectrum to be compared with the one predicted by the LSA. As expected, we find a good agreement with LSA for early times. Interestingly, for late times we obtain that the wave number of the maximum of the spectra tends to approach the deterministic value, $q_{m}$, corresponding to the LSA without stochasticity. Since the LSA with stochasticity also tends to $q_{m}$, we can conclude that 
the typical lengths of the patterns in advanced stages of the instability with stochasticity seem to be close to the length of maximum growth rate of the linear deterministic modes.

Encouraged by this result we also compare the LSA prediction with the experimental data from the instability of laser-melted copper films on a silicon oxide substrate. These data correspond to the early stages, where the holes start to grow, as well as to the stages of drop formation, i.e., after having passed through the processes of film breakup and dewetting. A special feature of these data is that they come from different spatial regions of the laser spot and thus received distinct illuminations. Thus, different times of a single evolution can be attributed to each region. These times were estimated here by fitting each experimental power spectrum to the corresponding LSA prediction. As a result, we found that the early stages of this experiment evolved with a noise that was almost white in space, while a strong spatial correlation appeared in the spectra for later times. Thus, correlated noise seems to be an important factor in the central regions of the laser spot, i.e., those with larger liquid lifetimes.

Taken together, our results provide a clear indication that the stochastic differential framework for metallic thin-film phenomena at the nanometric scale requires the inclusion of thermal noise with extended spatial correlations. We consider the present study only a first step towards the understanding of thermal noise in nonpolymeric films. We believe that our results justify further testing with more detailed experimental data and for a variety of film material.

\section{ACKNOWLEDGMENTS}

A.G.G. and J.A.D. acknowledge support from Consejo Nacional de Investigaciones Científicas y Técnicas de la República Argentina (CONICET, Argentina) with grant PIP 844/2011 and Agencia Nacional de Promoción de Científica y Tecnológica (ANPCyT, Argentina) with Grant No. PICT 931/2012. R.F. acknowledges the support of a Milstein scholarship (EXPTE.MINCYT 1141/14 and RES. 372/14), as well as the Universidad Nacional del Centro de la Provincia de Buenos Aires for hospitality during the completion of this work. The three authors thank the anonymous referees for their insightful comments that led us to improvements and clarifications in our presentation.

\section{APPENDIX: EIGENVALUES OF THE CORRELATION FUNCTION}

Here we calculate the eigenvalues of the Hilbert-Schmidt operator $\mathcal{Q}$ as defined by Eqs. (45) and (47). By using the variable $v=\pi u / L$, the eigenvalues can be written as

$$
\chi_{k}=\frac{A(\alpha, k)}{A(\alpha, 0)}
$$

where

$$
A(\alpha, k)=\int_{0}^{\pi} e^{-2 \alpha(\sin v)^{2}-2 i k v} d v
$$

and $\alpha$ is given by Eq. (49). In order to perform the above integral, we make the change of variables $2 v=\theta+\frac{\pi}{2}$, which leads to the following expression:

$$
\sin ^{2} v=\frac{1}{2}(1-\cos 2 v)=\frac{1}{2}(1+\sin \theta) .
$$

This one allows us to write Eq. (A2) in terms of $\sin \theta$, as

$$
\begin{aligned}
A(\alpha, k) & =\frac{1}{2} e^{-\alpha} e^{-l k \pi / 2} \int_{-\frac{\pi}{2}}^{\frac{3 \pi}{2}} e^{-\alpha \sin \theta} e^{-\imath k \theta} d \theta \\
& =\frac{(-l)^{k}}{2} e^{-\alpha} \int_{-\frac{\pi}{2}}^{\frac{3 \pi}{2}} e^{-\alpha \sin \theta} e^{-\imath k \theta} d \theta .
\end{aligned}
$$

The above substitution is convenient in view of the relation

$$
e^{\imath x \sin \theta}=\sum_{-\infty}^{\infty} e^{\imath m \theta} J_{m}(x),
$$

which becomes useful here on defining $x=i \alpha$. Thus, we have

$$
e^{-\alpha \sin \theta}=\sum_{-\infty}^{\infty} e^{\imath m \theta} J_{m}(l \alpha),
$$

where $J_{m}(\imath \alpha)$ is the Bessel function of order $m$. Now, we can also use the property

$$
J_{m}(\imath \alpha)=\imath^{m} I_{m}(\alpha)
$$

where $I_{m}(\alpha)$ is the modified Bessel function of order $m$. By replacing Eqs. (A5) and (A6) into Eq. (A3), we obtain

$$
A(\alpha, k)=\frac{(-l)^{k}}{2} e^{-\alpha} \sum_{-\infty}^{\infty} l^{m} I_{m}(\alpha) \int_{-\frac{\pi}{2}}^{\frac{3 \pi}{2}} e^{l(m-k) \theta} d \theta .
$$

Since the above integral yields $2 \pi \delta_{k m}$, we finally have

$$
A(\alpha, k)=\pi e^{-\alpha} I_{k}(\alpha),
$$

so the eigenvalue in Eq. (A1) becomes

$$
\chi\left(q_{k}\right)=\chi_{k}=\frac{I_{k}(\alpha)}{I_{0}(\alpha)},
$$

which is the expression in Eq. (48).
[1] L. Landau and E. Lifshitz, Fluid Mechanics (Permagon Press, Oxford, 1987).

[2] R. F. Fox and G. E. Uhlenbeck, Contributions to non-equilibrium thermodynamics. i. theory of hydrodynamical fluctuations, Phys. Fluids 13, 1893 (1970).

[3] K. T. Mashiyama and H. Mori, Origin of the Landau-Lifshitz hydrodynamic fluctuations in nonequilibrium systems and a new method for reducing the Boltzmann equation, J. Stat. Phys. 18, 385 (1978).
[4] D. Forster, D. R. Nelson, and M. J. Stephen, Long-Time Tails and the Large-Eddy Behavior of a Randomly Stirred Fluid, Phys. Rev. Lett. 36, 867 (1976).

[5] P. C. Hohenberg and J. B. Swift, Effects of additive noise at the onset of Rayleigh-Benard convection, Phys. Rev. A 46, 4773 (1992).

[6] J. B. Swift, Kenneth L. Babcock, and P. C. Hohenberg, Effects of thermal noise in taylor-couette flow with corotation and axial through-flow, Physica A 204, 625 (1994). 
[7] A. Oron, S. H. Davis, and S. G. Bankoff, Long-scale evolution of thin liquid films, Rev. Mod. Phys. 69, 931 (1997).

[8] K. Mecke and M. Rauscher, On thermal fluctuations in thin film flow, J. Phys.: Condens. Matter 17, S3515 (2005).

[9] M. Moseler and U. Landman, Formation, stability, and breakup of nanojets, Science 289, 1165 (2000).

[10] P. Hänggi and F. Marchesoni, Artificial brownian motors: Controlling transport on the nanoscale, Rev. Mod. Phys. 81, 387 (2009).

[11] U. Landman, Materials by numbers: Computations as tools of discovery, Proc. Natl. Acad. Sci. USA 102, 6671 (2005).

[12] S. Herminghaus, K. Jacobs, and R. Seemann, The glass transition of thin polymer films: Some questions, and a possible answer, Eur. Phys. J. E 5, 531 (2001).

[13] R. Seemann, S. Herminghaus, C. Neto, S. Schlagowski, D. Podzimek, R. Konrad, H. Mantz, and K. Jacobs, Dynamics and structure formation in thin polymer melt films, J. Phys.: Condens. Matter 17, S267 (2005).

[14] R. Seemann, S. Herminghaus, and K. Jacobs, Dewetting Patterns and Molecular Forces: A Reconciliation, Phys. Rev. Lett. 86, 5534 (2001).

[15] R. Fetzer, M. Rauscher, R. Seemann, K. Jacobs, and K. Mecke, Thermal noise influences fluid flow in thin films during spinodal dewetting, Phys. Rev. Let. 99, 114503 (2007).

[16] J. Becker, G. Grün, R. Seemann, H. Mantz, K. Jacobs, K. R. Mecke, and R. Blossey, Complex dewetting scenarios captured by thin-film models, Nat. Mat. 2, 59 (2003).

[17] H. Mantz, K. Jacobs, and K. Mecke, Utilizing minkowski functionals for image analysis: A marching square algorithm, J. Stat. Mech. 12, P12015 (2008).

[18] A. G. González, J. A. Diez, Y. Wu, J. D. Fowlkes, P. D. Rack, and L. Kondic, Instability of liquid cu films on a $\mathrm{SiO}_{2}$ substrate, Langmuir 29, 9378 (2013).

[19] K. Mecke G. Grün and M. Rauscher, Thin-film flow influenced by thermal noise, J. Stat. Phys. 122, 1261 (2006).

[20] Setsuo Ichimaru, Statistical Plasma Physics_Volume I: Basic Principles (Addison-Wesley, New York, 1992).

[21] H. Risken, The Fokker-Planck Equation (Springer, Berlin, 1989).
[22] J. Diez and L. Kondic, On the breakup of fluid films of finite and infinite extent, Phys. Fluids 19, 072107 (2007).

[23] Pao-Liu Chow, Stochastic Partial Differential Equations (Chapman \& Hall, New York, 2007).

[24] Lawrence C. Evans, An Introduction to Stochastic Differential Equations (American Mathematical Society, Washington DC, 2013).

[25] List of mathematical symbols, https://en.wikipedia.org/ wiki/List_of_mathematical_symbols.

[26] J. Diez, L. Kondic, and A. L. Bertozzi, Global models for moving contact lines, Phys. Rev. E 63, 011208 (2001).

[27] J. Diez and L. Kondic, Computing three-dimensional thin film flows including contact lines, J. Comp. Phys. 183, 274 (2002).

[28] W. H. Press, S. A. Teukolsky, B. P. Flannery and W. T. Vetterling, Numerical Recipes in Fortran (Cambridge University Press, New York, 1992).

[29] P. D. Rack, Y. F. Guan, J. D. Fowlkes, A. V. Melechko, and M. L. Simpson, Pulsed laser dewetting of patterned thin metal films: A means of directed assembly, Appl. Phys. Lett. 92, 223108 (2008).

[30] L. Kondic, J. Diez, P. Rack, Y. Guan, and J. Fowlkes, Nanoparticle assembly via the dewetting of patterned thin metal lines: Understanding the instability mechanism, Phys. Rev. E 79, 026302 (2009).

[31] Y. Wu, J. D. Fowlkes, P. D. Rack, J. A. Diez, and L. Kondic, On the breakup of patterned nanoscale copper rings into droplets via pulsed-laser-induced dewetting: Competing liquid-phase instability and transport mechanisms, Langmuir 26, 11972 (2010).

[32] Y. Wu, J. D. Fowlkes, N. A. Roberts, J. A. Diez, L. Kondic, A. G. González, and P. D. Rack, Competing liquid phase instabilities during pulsed laser induced self-assembly of copper rings into ordered nanoparticle arrays on $\mathrm{SiO}_{2}$, Langmuir, 27, 13314 (2011).

[33] J. D. Fowlkes, L. Kondic, J. Diez, and P. D. Rack, Self-assembly versus directed assembly of nanoparticles via pulsed laser induced dewetting of patterned metal films, Nano Lett. 11, 2478 (2011). 